\title{
Aiming for the complete utilization of sugar-beet pulp: Examination of the effects of mild acid and hydrothermal pretreatment followed by enzymatic digestion
}

\author{
Stefan Kühnel, Henk A Schols and Harry Gruppen*
}

\begin{abstract}
Background: Biomass use for the production of bioethanol or platform chemicals requires efficient breakdown of biomass to fermentable monosaccharides. Lignocellulosic feedstocks often require physicochemical pretreatment before enzymatic hydrolysis can begin. The optimal pretreatment can be different for different feedstocks, and should not lead to biomass destruction or formation of toxic products.

Methods: We examined the influence of six mild sulfuric acid or water pretreatments at different temperatures on the enzymatic degradability of sugar-beet pulp (SBP).

Results: We found that optimal pretreatment at $140^{\circ} \mathrm{C}$ of 15 minutes in water was able to solubilize $60 \% \mathrm{w} / \mathrm{w}$ of the total carbohydrates present, mainly pectins. More severe treatments led to the destruction of the solubilized sugars, and the subsequent production of the sugar-degradation products furfural, hydroxymethylfurfural, acetic acid and formic acid. The pretreated samples were successfully degraded enzymatically with an experimental cellulase preparation.

Conclusions: In this study, we found that pretreatment of SBP greatly facilitated the subsequent enzymatic degradation within economically feasible time ranges and enzyme levels. In addition, pretreatment of SBP can be useful to fractionate functional ingredients such as arabinans and pectins from cellulose. We found that the optimal combined severity factor to enhance the enzymatic degradation of SBP was between log $R_{0}^{\prime}=-2.0$ and $\log R_{0}^{\prime}=-1.5$. The optimal pretreatment and enzyme treatment solubilized up to $80 \%$ of all sugars present in the SBP, including $\geq 90 \%$ of the cellulose.
\end{abstract}

\section{Background}

Sugar-beet pulp (SBP) consists of up to $75 \% \mathrm{w} / \mathrm{w}$ of carbohydrates (dry matter). Arabinose, glucose and galacturonic acid (GA) are the main sugar moieties present in complex polysaccharide structures [1]. After sugar extraction, the pressed pulp has a dry-matter content of $18-23 \% \mathrm{w} / \mathrm{w}$. To date, SBP has been used mainly as animal feed or, in regions with no livestock farming, dumped in landfill $[2,3]$. The low dry-matter content makes combustion of SBP for heat and power production unfavorable; however, its low lignin and high sugar

\footnotetext{
* Correspondence: harry.gruppen@wur.nl
Laboratory of Food Chemistry, Wageningen University, Bomenweg 2, 6703

* Correspondence: harry.gruppen@wur.nl
Laboratory of Food Chemistry, Wageningen University, Bomenweg 2, 6703 HD Wageningen, the Netherlands
} (

content make it an interesting candidate for biorefinery and/or bioethanol production. The availability of new, pentose-fermenting yeast strains allows the efficient use of biomass for bioethanol production [4]; however, no yeast strains that allow the fermentation of uronic acids to ethanol are currently available.

For fermentation, the cell-wall material needs to be degraded into fermentable monosaccharides. To this end, lignocellulosic feedstocks are often structurally modified by a mild pretreatment before enzymatic release of fermentable monosaccharides [5]. The severity of this pretreatment can be measured by a combined severity factor $\left(\log \mathrm{R}_{0}{ }_{0}\right)$ that takes into account pretreatment time, pretreatment temperature, and acid concentration (w/w\%) 
and $\mathrm{pH}$ after pretreatment $[6,7,47]$. In general, a pretreatment has to be strong enough to disrupt the cellulosehemicellulose network and, if present, the cellulose-lignin network; however, as the severity of the pretreatment increases, more biomass is degraded to non-fermentable products and products toxic to yeast, such as furfural or hydroxymethylfurfural (HMF) [8]. Along with release of furfural and HMF, weak acids (such as acetic acid) which can have a negative effect on yeast growth, viability and fermentation, may also be released $[9,10]$. Although yeasts can adapt to moderate levels of furfural and detoxification, as shown previously [11], it is economically and ecologically favorable to avoid the formation of such compounds.

During the past decades, much work has been performed on the degradation of SBP, which has shown good yields of solubilized carbohydrates; however, in these studies, high levels of enzymes or chemicals were required [12-15]. To our knowledge, there are no data available for the degradation of SBP in commercially reasonable time spans and with economically feasible enzyme levels.

In this study, we examined the effect of hydrothermal or mild acid pretreatment on enhancing the enzymatic degradation of SBP. Several different pretreatments were used, with different temperatures and $\mathrm{pH}$ values. The pretreated samples were characterized and used for an enzymatic saccharification study to analyze the amounts of fermentable monosaccharides produced.

\section{Methods}

\section{Starting material}

SBP was obtained as frozen pressed pulp (23\% dry-matter content, harvest 2006; Suiker Unie, Dinteloord, The Netherlands). The pulp contained $68 \% \mathrm{w} / \mathrm{w}$ carbohydrates (dry weight). The constituent sugars were arabinose $(18 \% \mathrm{w} / \mathrm{w})$, glucose $(22 \% \mathrm{w} / \mathrm{w})$, uronic acids (UAs) $(18 \% \mathrm{w} / \mathrm{w})$, galactose $(5 \% \mathrm{w} / \mathrm{w})$, rhamnose $(2 \% \mathrm{w} / \mathrm{w})$, xylose $(2 \% \mathrm{w} / \mathrm{w})$ and mannose $(1 \% \mathrm{w} / \mathrm{w})$, respectively, and the pulp contained $4 \% \mathrm{w} / \mathrm{w}$ residual saccharose. Other components were protein $(8 \% \mathrm{w} / \mathrm{w})$, lipids and salts. Ferulic acid $(0.5 \% \mathrm{w} / \mathrm{w})$, acetic acid $(1.6 \% \mathrm{w} / \mathrm{w})$ and methanol $(0.4 \% \mathrm{w} / \mathrm{w})$ were present as ester-linked substituents of polysaccharides.

\section{Pretreatment}

Pretreatments were carried out with a dry-matter content of 5\% w/w: $152 \mathrm{~g}$ of pressed pulp was made up to $700 \mathrm{~g}$ with distilled water. The pulp was mixed for 5 minutes using a blender (type 7011G; Waring Commercial, Torrington, CT, USA) at low speed. Concentrated sulfuric acid $(180 \mu \mathrm{l})$ was added to the blended pulp, according to the conditions used (Table 1). Pretreatment was performed in a pressure reactor (Model
Table 1 Pretreatment conditions of sugar-beet pulp

\begin{tabular}{|c|c|c|c|c|c|}
\hline Sample & Temp, ${ }^{\circ} \mathrm{C}$ & $\mathrm{H}_{2} \mathrm{SO}_{4}{ }^{\mathrm{a}}$ & $\mathrm{pH}^{\mathrm{b}}$ & $\mathrm{R}_{0}^{\prime \prime}{ }^{\mathrm{c}}$ & $\log R^{\prime \prime}{ }_{0}^{d}$ \\
\hline $0-0$ & 0 & 0 & 5.3 & -1.77 & -6.97 \\
\hline $120-0$ & 120 & 0 & 4.8 & 1.76 & -3.04 \\
\hline $140-0$ & 140 & 0 & 4.4 & 2.35 & -2.05 \\
\hline $170-0$ & 170 & 0 & 4.1 & 3.24 & -0.86 \\
\hline $120-1$ & 120 & $1^{*}$ & 3.9 & 2.11 & -2.14 \\
\hline $140-1$ & 140 & $1^{*}$ & 3.9 & 2.70 & -1.65 \\
\hline $170-1$ & 170 & $1^{*}$ & 3.8 & 3.62 & -0.53 \\
\hline
\end{tabular}

${ }^{\mathrm{a} S u l f u r i c}$ acid concentration given in $\% \mathrm{w} / \mathrm{w}$ of the dry-matter content.

${ }^{\mathrm{b}} \mathrm{pH}$ values of the solutions after pretreatment.

'Combined severity factor according to Overend et al. [47].

${ }^{d}$ Decade logarithm of the combined severity factor; $1 \% \mathrm{w} / \mathrm{w}$ of the drymatter content corresponds to a sulfuric acid concentration of $0.05 \mathrm{w} / \mathrm{v} \%$.

4520; Parr Instrument Company, Moline, IL, USA) connected to a power controller (Model 4875; Parr Instrument Company) and operated by a process controller (Model 4875; Parr Instrument Company). The reactor was equipped with an oil bath $\left(200^{\circ} \mathrm{C}\right)$ that caused oil to flush through a spindle inside the reactor. An electric heating cord was attached to the outside of the reactor to accelerate the average heating rate and to maintain a constant temperature after heating. The oil bath was turned off when a temperature $20^{\circ} \mathrm{C}$ below the desired temperature was reached in the reactor. After 12 minutes, the electric heater was removed, and the reactor was cooled by flushing tap water $\left(\right.$ at $\left.10^{\circ} \mathrm{C}\right)$ in a specially designed flow-through water bath, resulting in an overall pretreatment time of 15 minutes at the pretreatment temperature chosen. Temperature and pressure were recorded, and the $\mathrm{pH}$ of the samples was determined before and after the pretreatment. Directly after the pretreatment, $1 \mathrm{ml}$ of supernatant was removed and filtered through a 0.2 $\mu \mathrm{m}$ round filter (Whatman plc, Maidstone, Kent, UK) to determine the free amounts of lactic acid, acetic acid, formic acid, furfural and hydroxymethyl furfural. The severities of the pretreatments were calculated as the combined severity factor $[6,7,47]$ :

$$
\mathrm{R}_{0}^{\prime}\left(\mathrm{R}_{0}^{\prime}=\mathrm{t} \times\left(10^{-\mathrm{pH}}\right) \times \exp \times((\mathrm{T}-100) / 14.75)\right) \text {, }
$$

and expressed as $\log \mathrm{R}_{0}{ }_{0}$ according to Kabel et al. [16]). This factor is an indicator for the severity of a pretreatment, and takes into account the pretreatment temperature, time, acid concentration and final $\mathrm{pH}$.

\section{Mass balance}

The process of sample separation and mass-balance determination is summarized in Figure 1. Nylon pantyhose (15 denier) were used to filter the pretreated slurries. The remaining solids were wrapped in a double layer of cheesecloth, and pressed in a hydraulic press (IKA Werke GmbH \& Co. KG, Staufen, Germany) at 100 bar until draining ceased. The pressed pellet was 


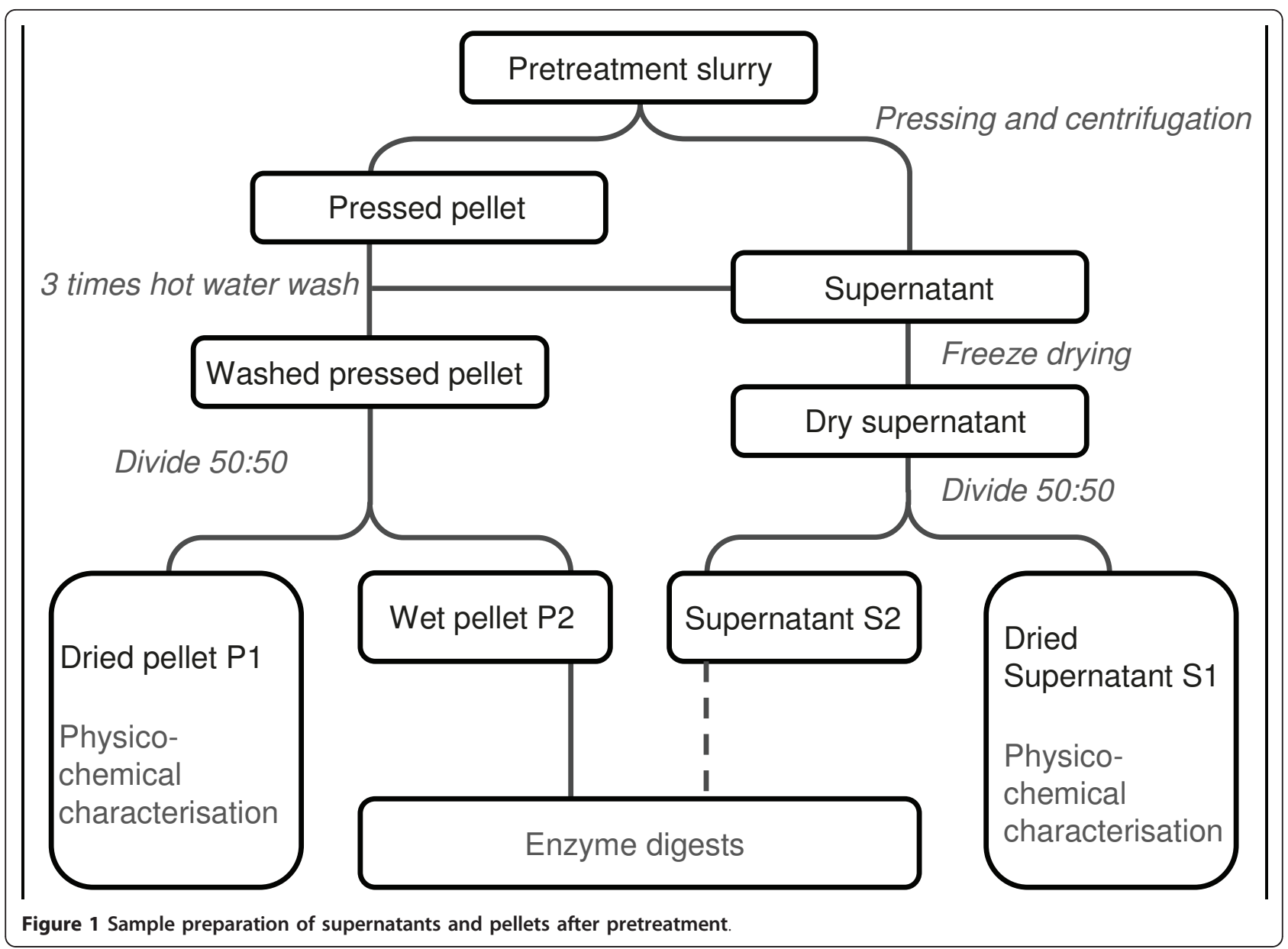

soaked three times with $500 \mathrm{ml}$ distilled water at $60^{\circ} \mathrm{C}$ for 10 minutes, and pressed again. The supernatant was weighed and subsequently combined with the washes. The supernatant and pellets were both divided in two, and one half (S1 and P1) of each was freezedried for chemical characterization, and the other half (S2 and P2) was frozen for enzymatic digestion. The P1 fractions were weighed before and after freeze-drying, and the dry-matter content was determined as an indicator of the water-binding capacity after pretreatment. The S1 supernatants collected after pressing were spun in a centrifuge $(12,320 \times \mathrm{g})$ at $4^{\circ} \mathrm{C}$ for 20 minutes to remove cell debris. The clear S1 supernatants were freeze-dried, and the debris was added to the P1 pellet fraction. The S2 supernatants were concentrated to their original concentrations, and stored frozen $\left(-20^{\circ} \mathrm{C}\right)$.

\section{Dry-matter content}

Freeze-dried samples (20-40 mg) were weighed into aluminum cups (diameter $50 \mathrm{~mm}$ ) and dried in an oven at $70^{\circ} \mathrm{C}$ for up to 3 days, until the weight was constant.
Chemical characterization of pretreated SBP

After freeze-drying, the P1 pellets were weighed and ground $(4$ minutes at $100 \mathrm{~Hz}$ ) in a swinging mill (Model M2200; Retsch GmbH, Haan, Germany) using $25 \mathrm{ml}$ stainless steel containers with two stainless-steel metal balls (diameter $10 \mathrm{~mm}$ ) per container.

The freeze-dried S1 supernatants were treated in the same way except for the supernatants S1 0-0, S1 170-0 and S1 170-1, which did not crystallize but instead formed a syrup. For these fractions, the syrup was dissolved in distilled water, and the volume was determined. An aliquot $(1 \mathrm{ml})$ was freeze-dried and subsequently oven-dried to calculate the dry-matter content of the solutions. The different crystallization behavior did not affect the subsequent results.

\section{Enzyme digestion \\ Activity assays}

Glycosidase activities in the experimental enzyme preparation C1-G1 were measured by determining the hydrolysis of $p$-nitrophenyl- $\beta$-d-glucopyranoside, $p$ nitrophenyl- $\beta$-d-xylopyranoside or $p$-nitrophenyl- $\beta-1$ - 
arabinofuranoside at $37^{\circ} \mathrm{C}$ after 10 minutes of incubation. The reaction mixture consisted of $25 \mu$ l substrate solution $(4 \mathrm{mg} / \mathrm{ml})$ and $10 \mu \mathrm{l}$ of the suitable enzyme concentration in $50 \mathrm{mmol} / \mathrm{l}$ sodium acetate buffer $\mathrm{pH}$ 5.0. The reaction was stopped by adding $40 \mu \mathrm{l}$ of reaction mixture to $60 \mu \mathrm{l}$ of $250 \mathrm{mmol} / \mathrm{l}$ TRIS-HCl buffer $\mathrm{pH}$ 8.8. The color formation was measured at $410 \mathrm{~nm}$ in a microtiter plate reader. One unit of activity was defined as $1 \mu \mathrm{mol}$ of glucose, xylose or arabinose liberated per minute under the conditions specified. The molar extinction coefficient under these assay conditions was $0.00158 / \mu \mathrm{mol} / \mathrm{l} / \mathrm{mm}$. Xylanase and cellulase activities were determined by measuring the amount of reducing sugars released from the hydrolysis of mediumviscosity wheat arabinoxylan (Megazyme International Ireland Limited, Bray, Wicklow, Ireland) and phosphoric acid-treated cellulose [17]. The reaction mixture consisted of $50 \mu \mathrm{l}$ substrate solution $(15 \mathrm{mg} / \mathrm{ml})$ and $20 \mu \mathrm{l}$ of the suitable enzyme concentration in $60 \mathrm{mmol} / \mathrm{l}$ sodium acetate buffer $\mathrm{pH}$ 5.0. The reaction mixtures were preheated at $37^{\circ} \mathrm{C}$ before the enzyme was added. After an incubation time of 10 minutes, $25 \mu$ l of the reaction mixture were used to determine the amount of reducing sugars using a PAra-Hydroxy Benzoic Acid Hydrazide (PAHBAH) assay [18].

The activities of polygalacturonase, rhamnogalacturonase, arabinanase and galactanase were determined by measuring the amount of reducing sugars released from the hydrolysis of poly-GA (Fluka, Sigma-Aldrich, Schnelldorf, Germany), linear arabinan (Megazyme) and potato galactan (Megazyme).The reaction mixture consisted of $180 \mu \mathrm{l}$ substrate $(5 \mathrm{mg} / \mathrm{ml})$ in $50 \mathrm{mmol} / \mathrm{l}$ sodium acetate buffer $\mathrm{pH} 5.0$, which was preheated to $37^{\circ} \mathrm{C}$ before $20 \mu \mathrm{l}$ of the relevant enzyme concentration were added. The reaction mixture. After an incubation time of 20 minutes, $10 \mu \mathrm{l}$ of the reaction mixture were used to determine the amount of reducing sugars using the PAHBAH assay [19]. Calibration curves of the different monosaccharides (50 to $750 \mu \mathrm{g} / \mathrm{ml}$ ) were used for quantification.

\section{Digestions of pretreated pulp}

Pressed pulp P2 (5\% w/w, $0.2 \%$ of the initial dry-matter) was digested with $10 \mu \mathrm{l}$ of an experimental enzyme mixture (protein content $75 \mathrm{mg} / \mathrm{ml}, 1 \% \mathrm{w} / \mathrm{w}$ enzyme level) (C1-G1; Dyadic Netherlands, Wageningen, The Netherlands) in $100 \mathrm{mmol} / \mathrm{l}$ sodium acetate buffer $\mathrm{pH} 5.5$ for $2,5,10,24$ or 48 hours. C1-G1 is an enzyme preparation of the fungus Chrysosporium lucknowense [20], which is rich in cellulase and xylanase activities, but also contains arabinanase and galactanase activities (Table 2). Ampicillin $100 \mu \mathrm{g} / \mathrm{ml}$ was added to prevent microbial growth. The reactions were carried out in $2 \mathrm{ml}$ microcentrifuge tubes placed in an microcentrifuge tube shaker kept at $40^{\circ} \mathrm{C}$ and shaken at $800 \mathrm{rpm}$. Enzymes were
Table 2 Activity profile of enzyme mix C1-G1

\begin{tabular}{lr}
\hline Activity & U/mg protein \\
\hline$\beta$-Glucosidase & 0.198 \\
$\beta$-Xylosidase & 0.012 \\
$\alpha$-Arabinofuranosidase & 0.035 \\
Xylanase & 1.322 \\
Cellulase & 1.400 \\
Arabinanase & 0.100 \\
Galactanase & 0.142 \\
Polygalacturonase/pectate lyase & 0.006 \\
\hline
\end{tabular}

inactivated by placing in a waterbath at $100^{\circ} \mathrm{C}$ for 15 minutes. The samples were spun in a centrifuge for 10 minutes at $20,000 \times \mathrm{g}$ and $20^{\circ} \mathrm{C}$, and $200 \mu \mathrm{l}$ of the supernatant was used for high-performance size exclusion chromatography (HPSEC) and high-performance anion exchange chromatography (HPAEC) analysis. The sugar composition of the remaining pellets was analyzed by gas chromatography with flame ionization detection (GC-FLD) after alditol acetate derivatization.

\section{Chromatography}

\section{Sugar composition}

The neutral sugar composition was determined by analyzing the sugars as their alditol acetate derivatives using GC-FLD. A gas chromatograph (Thermo Focus; Thermo Scientific, Waltham, MA, USA) and autosampler (Focus AS 3000; Thermo Scientific) equipped with a column $(15 \mathrm{~m} \times 0.53 \mathrm{~mm}, 1 \mu \mathrm{m}$ film; DB-225; J\&W Scientific, Santa Clara, IL, USA) was used. The samples were hydrolyzed stepwise with $72 \% \mathrm{w} / \mathrm{w}$ sulfuric acid for 1 hour at $30^{\circ} \mathrm{C}$, followed by $1 \mathrm{M}$ sulfuric acid for 3 hours at $100^{\circ} \mathrm{C}$. Subsequently, the free monosaccharides were reduced and derivatized to their corresponding alditol acetates [21]. The samples were dissolved in $0.4 \mathrm{ml}$ acetone, and $1 \mu \mathrm{l}$ was injected with a pre-injection dwell time of 1 second and a post-injection dwell time of 2 seconds. The sample was then eluted using the following temperature profile: 0 to 2 minutes at $180^{\circ} \mathrm{C}, 2$ to 17 minutes at a linear gradient from 180 to $210^{\circ} \mathrm{C}$, and 17 to 22 minutes at $210^{\circ} \mathrm{C}$.

UA content was determined colorimetrically [22] with 3-phenylphenol using an automated analyzer (Skalar Analytical, Breda, The Netherlands). A GA standard curve $(12.5$ to $100.0 \mu \mathrm{g} / \mathrm{ml})$ was used for quantification.

\section{Organic-acid content}

The content of organic acids, HMF, furfural and methanol was analyzed by high performance liquid chromatography with a (Ultimate 3000; Dionex Corp., Sunnyvale, CA, USA) equipped with an ion-exclusion column (300 $\mathrm{mm} \times 7.8 \mathrm{~mm}$; Aminex HPX-87H; Bio-Rad Laboratories, Hercules, CA, USA) in combination with a selfpacked guard column $(50 \mathrm{~mm} \times 7.8 \mathrm{~mm}$; AG 50W-X4 
resin; Bio-Rad Laboratories). Samples were eluted with 5 $\mathrm{mmol} / \mathrm{l}$ sulfuric acid at a flow rate of $0.6 \mathrm{ml} / \mathrm{min}$ at $30^{\circ}$ C. The S1 supernatants were filtered through a $0.2 \mu \mathrm{m}$ round filter (Whatman), and $20 \mu \mathrm{l}$ samples used for injection. The samples were quantified by single-component standards of known concentrations $(0.5 \mathrm{mg} / \mathrm{ml})$. Elution was monitored by refractive index detection (Shodex RI 101; Showa Denko K.K., Kawasaki, Japan).

\section{Monomer and oligomer analysis}

The monosaccharide and oligosaccharide levels of the digests were analyzed by HPAEC with pulsed amperometric detection (PAD) (ITS-3000 HLPC; Dionex) equipped with an analytical column $(2 \mathrm{~mm} \times 250 \mathrm{~mm}$, CarboPac PA1; Dionex) in combination with a guard column $(2 \mathrm{~mm} \times 50 \mathrm{~mm}$, CarboPac PA1; Dionex $)$ at a flow rate of $0.3 \mathrm{ml} / \mathrm{min}$. Arabinose, rhamnose, galactose, glucose, xylose, mannose and GA monosaccharides in the range from 2 to $30 \mu \mathrm{g} / \mathrm{ml}$ were used for quantification. Oligomeric standards included arabinose oligomers (degree of polymerization (DP) 2 to $6,10 \mu \mathrm{g} / \mathrm{ml}$; Megazyme), GA oligomers (DP 2 and DP 3, $10 \mu \mathrm{g} / \mathrm{ml}$ ), a potato galactan digest containing galactose oligomers (DP 2 to $5 ; 5 \mathrm{mg} / \mathrm{ml}$ potato galactan; Megazyme) partially digested with Aspergillus niger endogalactanase (purified in our laboratory) and cellodextrins (DP2 to 7; kind gift of Dr Vladimir Farkas, Institute of Chemistry, Slovak Academy of Sciences, Bratislava, Slovakia). The samples $(10 \mu \mathrm{l} ; 50$ to $100 \mu \mathrm{g} / \mathrm{ml})$ were eluted in post column addition mode with the following elution profile: 0.0 to 30.0 minutes in water, 30.0 to 30.1 minutes in 0.0 to $0.1 \mathrm{~mol} / \mathrm{l} \mathrm{NaOH}, 30.1$ to 32 in $0.1 \mathrm{M} \mathrm{NaOH}$, 32.0 to 62.5 minutes in 0.0 to $0.4 \mathrm{~mol} / \mathrm{l} \mathrm{NaOAc}$ in 0.1 $\mathrm{mol} / \mathrm{l} \mathrm{NaOH}, 62.5$ to 67.0 minutes in $1.0 \mathrm{~mol} / \mathrm{l} \mathrm{NaOAc}$ dissolved in $0.1 \mathrm{~mol} / \mathrm{l} \mathrm{NaOH}, 67.0$ to 75.0 minutes in $0.1 \mathrm{~mol} / \mathrm{l} \mathrm{NaOH}$, and 75.1 to 90.0 minutes in water (equilibration). $\mathrm{NaOH}(0.5 \mathrm{~mol} / \mathrm{l}, 0.1 \mathrm{ml} / \mathrm{min})$ was added via a post column to allow PAD detection from 0.0 to 30.1 minutes and from 68.5 to 90.0 minutes.

\section{Ferulic acid analysis}

The amount of ester-linked ferulic acid was determined after alkaline hydrolysis and ethyl ether extraction as described previously [23] using reversed phase ultrahigh-performance liquid chromatography (UHPLC) on a UHPLC system (Accela; Thermo Scientific) equipped with a column $(2.1 \mathrm{~mm} \times 150 \mathrm{~mm}, 1.9 \mu \mathrm{m}$ particle size; Hypersyl GOLD; Thermo Scientific). Elution was monitored with the built-in polydiode array multi-wavelength detector set at $335 \mathrm{~nm}$.

\section{Molecular mass distribution}

HPSEC was performed on a system (Ultimate 3000; Dionex) equipped with four columns (TSK-Gel superAW; Tosoh Bioscience, Tokyo, Japan) in series: a guard column $(6 \mathrm{~mm} \times 40 \mathrm{~mm})$ and three separation columns (4000, 3000 and 2500; $6 \mathrm{~mm} \times 150 \mathrm{~mm})$. The samples
$(10 \mu \mathrm{l}, 5 \mathrm{mg} / \mathrm{ml})$ were eluted with $0.2 \mathrm{~mol} / \mathrm{l}$ sodium nitrate at $40^{\circ} \mathrm{C}$ at a flow rate of $0.6 \mathrm{ml} / \mathrm{min}$. Molecular masses were estimated with the help of molecular mass standards (Pullulan; Polymer Laboratories, Varian Inc., Palo Alto, CA, USA). Elution was monitored by refractive index detection (Shodex RI 101) and UV detection (internal detector) at four wavelengths $(\lambda=235 \mathrm{~nm}, 310$ $\mathrm{nm}, 325 \mathrm{~nm}$ and $345 \mathrm{~nm}$ ). The relative peak area distribution was calculated by the integration of the peak area in three time increments. The total peak area was calculated as the total peak area from 7.0 to $18.0 \mathrm{~min}$ utes without the negative peak (15.5 minutes) plus the peak area difference between the negative peak of a water blank and the negative peak of the supernatant sample.

\section{Protein content}

The protein content of the pretreated samples was determined by combustion (the Dumas method) with a nitrogen analyzer (FlashEA 1112 series; Thermo Scientific) in accordance with the manufacturer's instructions, using methionine as a standard and a nitrogen factor of 6.25 .

\section{Results and Discussion \\ Mass of pretreated SBP}

The effects of the different pretreatments on the solubilization of SBP were evaluated by weighing on a mass balance, taking into account the respective amounts of solubilized, residual and decomposed material. The combined severity factors (from the equation given in Methods) of the pretreatments are given in Table 1. The mass distribution of the samples after pretreatment was plotted against $\log \mathrm{R}_{0}$ (Figure 2). A $\log \mathrm{R}_{0}{ }_{0}$ of around -3.0 to -1.5 was necessary to solubilize substantial parts of the beet pulp. With increasing severity of the pretreatment, an increasing amount of sample was lost, probably by chemical destruction of carbohydrates (for example, Maillard reaction) and formation of volatile compounds. Such formation of volatile compounds was seen during pretreatments with $\log \mathrm{R}_{0} \geq-0.86$ (samples 170-1 and 170-0), for which a temperature-independent pressure increase was observed after pretreatment (see Additional file 1, Figure S1). A strong color change after pretreatment and a caramel-like aroma indicated maillardation under the conditions used (data not shown).

Generally, more material was solubilized with increasing pretreatment severity, but a loss of mass was seen for all samples. These losses were mainly attributable to the mechanical loss of material during blending and sample handling. When this mechanical loss was averaged over the first five pretreatments, it was estimated to account for roughly $8 \% \mathrm{w} / \mathrm{w}$. Pretreatments with $\log$ $\mathrm{R}_{0}{ }_{0} \geq-0.86$ (sample 140-1) led to major losses in mass, probably due to chemical decomposition, and no further 


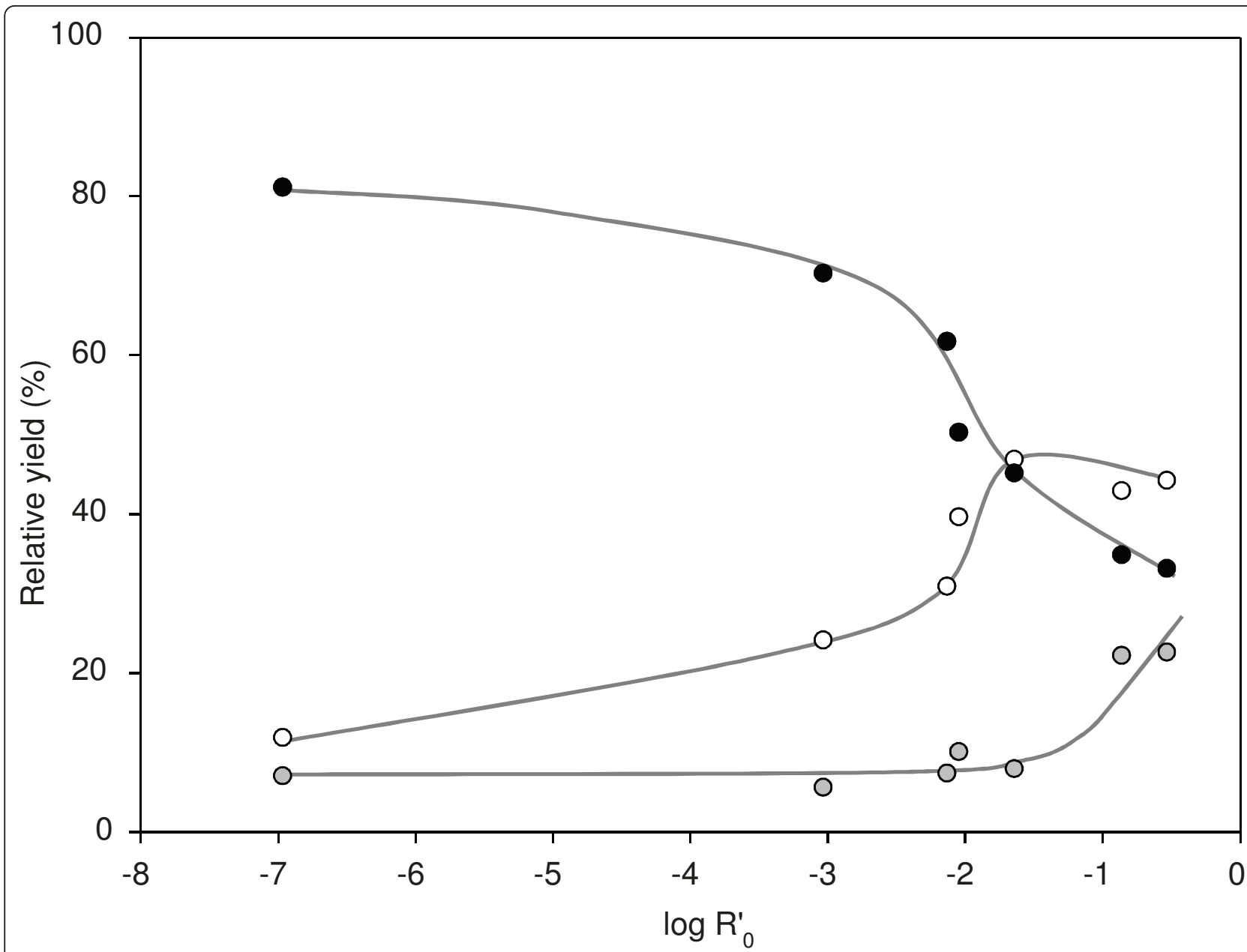

Figure 2 Distribution of pretreated sugar-beet pulp over supernatant fractions, pellet fractions and decomposed material in relation to the combined severity factor $\left(\log R^{\prime} 0\right)$. Black dots = pellets, white dots = supernatants, grey dots = decomposed material. Lines indicate trends.

increase in the level of soluble compounds in the supernatant (Figure 2). The pellet and supernatant curves were both sigmoidally shaped, which could imply that the range between solubilization and chemical degradation of the pulp is narrow, and hence requires good process control. If more severe treatments are required for effective disruption of the cellulose network, solubilized temperature-labile compounds should first be removed from the reactor to minimize their destruction. Such removal of soluble compounds could be carried out by using a flow-through reactor setting or an extruder.

\section{Sugar composition of released and residual carbohydrate material}

The predominant sugars released were the pectin-associated sugars arabinose, galactose and GA, and there were also some traces of rhamnose, derived from the rhamnogalacturonan part of pectin (Figure $3 \mathrm{~A}$ ). In contrast to the increasing levels of solubilized pectin- derived sugars, around $3 \% \mathrm{w} / \mathrm{w}$ of the glucose component was released irrespective of the severity of the pretreatment. This released glucose was probably derived from the residual saccharose in the pressed pulp. When pretreated at $170^{\circ} \mathrm{C}\left(\log \mathrm{R}_{0}{ }_{0} \geq-0.86\right)$, the arabinose and GA yields in the supernatant fraction decreased, whereas the levels of the others sugars remained constant or increased further. Decomposition levels of $40 \%$ and $90 \%$ were calculated for arabinose and GA, respectively, suggesting that arabinose and GA are both heat-labile (Figure 3D). Pectins have been shown to decarboxylate at high temperatures and high acid levels [24], leading to the formation of furfural [25] and/or arabinose [26]. Therefore, the apparent arabinose levels we detected might be a result of arabinose being lost during pretreatment and new arabinose chains being formed during the decomposition of GA.

High solubilization of arabinan and galacturonan from SBP after exposure to temperatures of 150 to $175^{\circ} \mathrm{C}$ has 

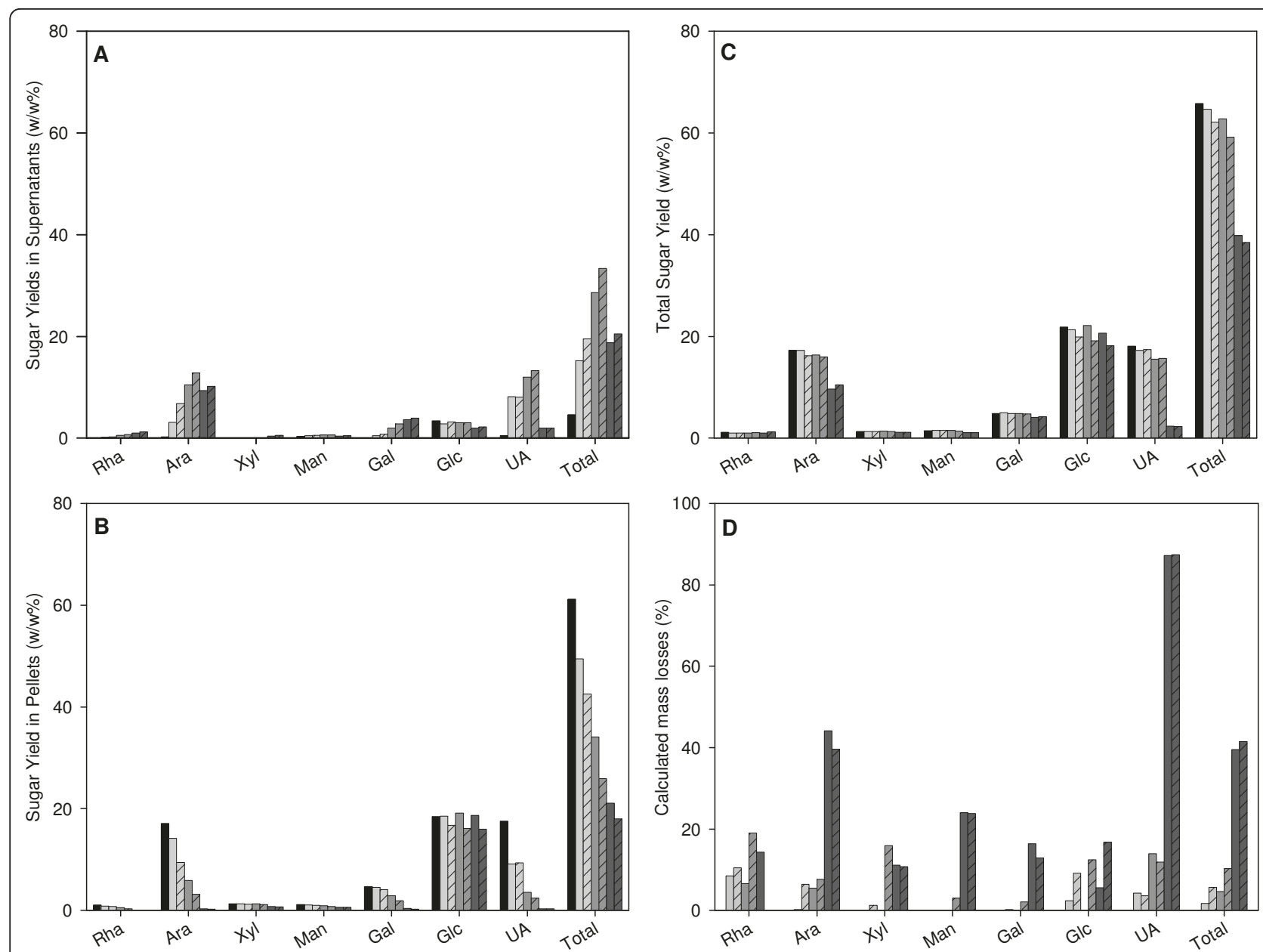

Figure 3 Sugar yields of pretreated sugar-beet pulp fractions. The data are expressed as percentage w/w based of starting material before pretreatment. Sugar yields of (A) the supernatant fractions, (B) the pellet fractions, (C) total pretreated material (sum of (A) + (B), (D) Calculated individual sugar losses during pretreatment. Black bars $=$ untreated pulp, light-grey bars $=120^{\circ} \mathrm{C}$ fractions, grey bars $=140^{\circ} \mathrm{C}$ fractions, dark-grey bars $=170^{\circ} \mathrm{C}$ fractions, shaded bars $=$ pretreatments with $1 \% \mathrm{~W} / \mathrm{W}$ sulfuric acid (on a dry-matter basis), non-shaded bars = pretreatments in water.

been reported previously [14,27]. Temperatures above $160^{\circ} \mathrm{C}$ led to decreasing recovery of GA, whereas arabinose recovery was stable up to $167^{\circ} \mathrm{C}$ [27], and most of the cellulose remained insoluble under the conditions used. Our data are in line with these observations.

We found that the sugar yields in the pellets decreased in line with the increasing severity of pretreatment (Figure 3B). Approximately $30 \%$ of the total sugars present in the starting material were left in the pellet fraction of sample 170-1. The absolute amounts of glucose remained almost constant, so that the relative glucose content of the pellet fractions increased up to $90 \%$ (moles) (see Additional file 2, Table s1). Even though cellulose was not solubilized during pretreatment, the solubilization of arabinan might also have had a positive effect on the enzymatic degradation of cellulose. It has been shown previously that arabinan can absorb to cellulose in vitro [28], thus removal of arabinan would be expected to lead to greater exposure of the cellulose surface to enzymes.

When the yields of the individual sugars present in the pellet and supernatant fractions were summed (Figure 3C), a decrease was seen for the pretreatments with $\log R_{0}^{\prime} \geq-0.86$ (samples 170-0 and 170-1), which was similar to the yield of the total material after pretreatment (Figure 2).

\section{Sugar-degradation products formed during pretreatment}

Besides lowering the yields, biomass pretreatment carries the risk of forming undesired degradation products that could negatively influence later use, such as in yeast fermentation for bioethanol production. We therefore investigated the samples to identify formation of formic acid, acetic acid, lactic acid, furfural and HMF (Figure 4). 


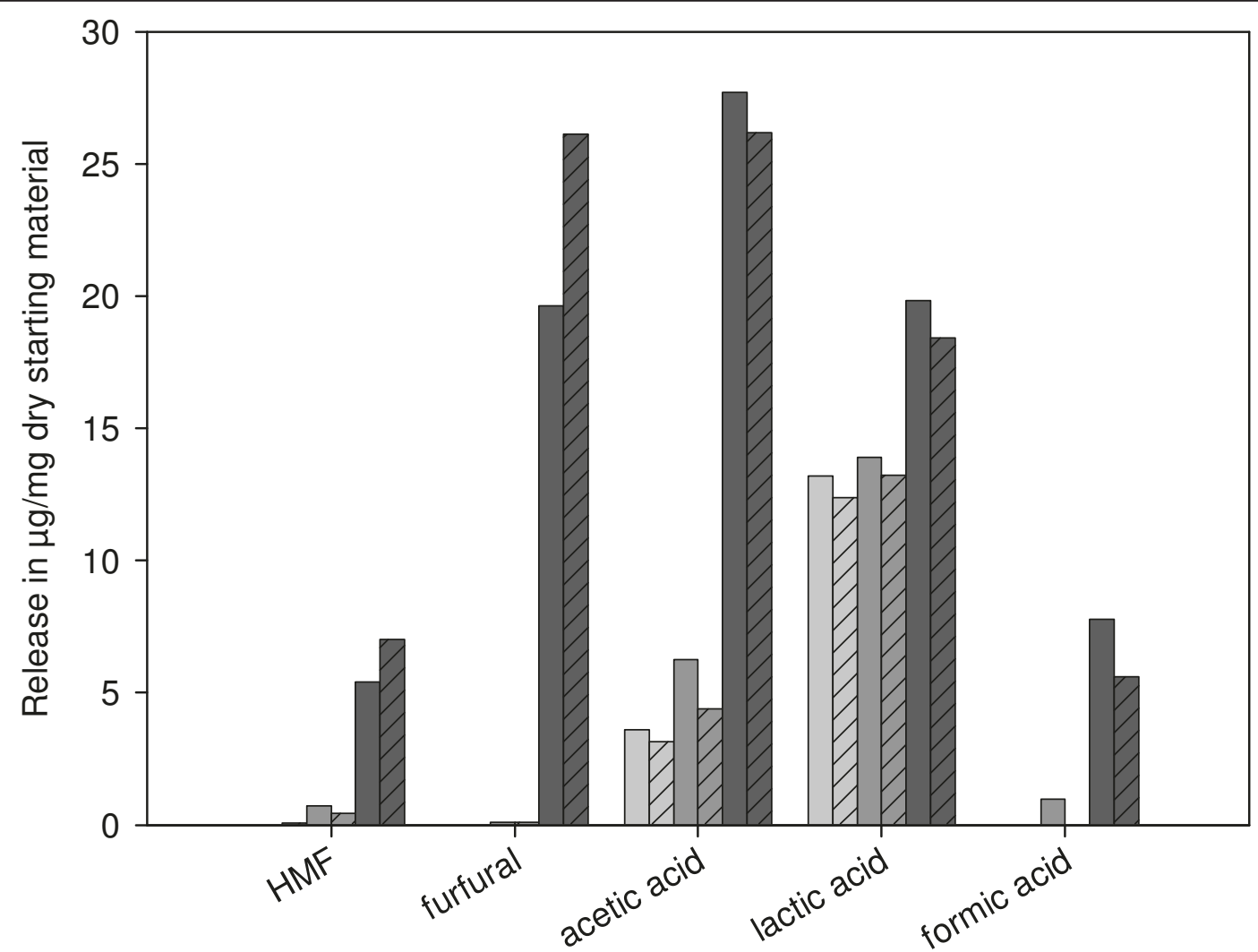

Figure 4 Specific sugar-degradation products formed after pretreatment. Light-grey bars $=120^{\circ} \mathrm{C}$ pretreatment, grey bars $=140^{\circ} \mathrm{C}$ pretreatment, dark-grey bars $=170^{\circ} \mathrm{C}$ pretreatments, shaded bars $=$ pretreatments with $1 \% \mathrm{w} / \mathrm{w}$ sulfuric acid (on a dry = matter basis), non $=$ shaded bars $=$ pretreatments in water.

HMF, furfural and formic acid were only seen for pretreatments with $\log \mathrm{R}_{0} \geq-0.86$ (samples $170-0$ and 170 1 ), which were both treated at $170^{\circ} \mathrm{C}$. HMF formation in these samples may be linked to the thermal decomposition of fructose, which derives from the autohydrolysis of saccharose. It has been shown previously that fructose is more acid-labile and five times more reactive than glucose [29], probably due to a higher level of open-ring conformation at higher temperatures [30]. HMF formation for the samples $170-0(0.55 \% \mathrm{w} / \mathrm{w}, 0.04 \mu \mathrm{mol})$ and $170-1(0.71 \% \mathrm{w} / \mathrm{w}, 0.06 \mu \mathrm{mol})$ may be a result of the degradation of 33 to $50 \%$ of the saccharose-derived fructose $(2 \% \mathrm{w} / \mathrm{w}, 0.12 \mu \mathrm{mol}$ in SBP).

Furfural formation was linked to pentose and UA degradation [25,31], and only occurred in samples 170-0 $(2.1 \% \mathrm{w} / \mathrm{w}, 0.2 \mu \mathrm{mol})$ and $170-1(2.6 \% \mathrm{w} / \mathrm{w}, 0.25 \mu \mathrm{mol})$. This could explain 14 to $18 \%$ of the arabinose degradation (around $1.4 \mu \mathrm{mol}$ in the pulp) or 20 to $25 \%$ of the UA degradation (around $1 \mu \mathrm{mol}$ ) we observed. Because $40 \%$ of the arabinose and $90 \%$ of the GA were decomposed (Figure 3D), it is likely that destruction of arabinose and GA also occurs via different mechanisms or yields different end-products.
For example, pectins can undergo $\beta$-elimination at high temperatures to form unsaturated GA and unsaturated GA oligomers [32]. A combination of $\beta$-elimination and decarboxylation, resulting in a pyranose ring structure of unsaturated 4,5-1-arabinopyranose and carbon dioxide, has also been proposed[33]. Carbon dioxide could be responsible for the temperature-independent pressure increase we saw during the $170^{\circ} \mathrm{C}$ pretreatments (see Additional file 1, Figure S1).

Formation of formic acid may be linked to further degradation of furfural and HMF at higher temperatures [31]. Furfural and HMF may also polymerize to form humins under acidic conditions and higher temperatures [34]; however, humin formation cannot explain the mass loss we found at $\log \mathrm{R}_{0} \geq-0.86$. In addition, it generally requires higher acid concentrations [34]. The presence of acetic and lactic acid in all our pretreated samples may also indicate contamination of the SBP starting material with acid-releasing bacteria. The release of acetic and lactic acid strongly increased at $\log \mathrm{R}_{0}$ $\geq-0.86$. The acetic acid release (up to $2.5 \% \mathrm{w} / \mathrm{w}$ ) can partly be explained by pectin deacetylation, because SBP contains around $1.6 \% \mathrm{w} / \mathrm{w}$ ester-linked acetic acid. In 
addition, acetic and lactic acid were also formed during the Maillard reaction.

The data from these experiments suggest that pretreatments at $\log \mathrm{R}_{0}{ }_{0} \geq-0.86$ led to the enhanced destruction of arabinose and GA and to the production of toxic compounds that could negatively affect fermentation.

\section{Compressibility of pretreated pulp}

The compressibility was studied as an indicator of the water-binding capacity (WBC) of the SBP. Water binding in plant cell walls of dicotyledons and non-graminaceous monocotyledons is usually attributable to pectin [35]. The pellets obtained after pretreatment had reduced levels of pectic arabinan and galacturonan (Figure 3B), but not all had reduced WBC (Table 3). The pellets of the $120^{\circ} \mathrm{C}$ and $140^{\circ} \mathrm{C}$ samples, by contrast, retained up to $207 \%$ more water than the untreated pulp. The $140^{\circ} \mathrm{C}$ pretreated samples had a water content $50 \%$ higher than the untreated pulp, whereas their pectin content was decreased by 70 to $80 \%$ (Figure 3B). The $170^{\circ} \mathrm{C}$ pretreated pellets were almost completely devoid of pectic sugars (Figure 3B), and after pressing, they retained only half the amount of water that the pressed untreated pulp retained. Hence, the swelling behavior of SBP is not only related to its pectin content, but also depends on other parameters, such as the cellwall architecture.

Increased swelling and $\mathrm{WBC}$ have been described previously for pellets of alkali-extracted beet fibers [36,37], even though these fibers had strongly reduced levels of pectic arabinan and galacturonan. Analogously, it has been reported for the cell-wall material of wheat flour that enzymatic removal of arabinoxylan increased the WBC of the residual, cellulose-rich material [38]. Possible explanations for the increased water binding include the total polysaccharide content, charge, crystallinity of the cellulose, specific surface area, and hydrophobic components [37].

These results indicate that dry-matter contents higher than $5 \%$ may not be possible for pretreatment and enzymatic digestion of SBP, because it is not possible to pump or stir the pulp efficiently. Bioethanol or biogas

Table 3 Water-binding capacity of pretreated samples

\begin{tabular}{lrr}
\hline Sample1 & ${\text { Bound water, } \mathbf{~ g / g ~} \text { pellet }^{\mathbf{a}}}$ & Change, $^{\text {\% }}$ \\
\hline $0-0$ & 2.4 & 0 \\
$120-0$ & 5.5 & 133 \\
$120-1$ & 7.2 & 207 \\
$140-0$ & 3.3 & 42 \\
$140-1$ & 3.6 & 54 \\
$170-0$ & 1.4 & -41 \\
$170-1$ & 1.2 & -47 \\
\hline
\end{tabular}

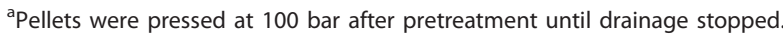

production requires higher substrate concentrations to make the downstream processing (for example, distillation/rectification) economically feasible. To allow higher dry-matter concentrations, the WBC could be reduced either enzymatically by the addition of an endopolygalacturonase or thermophysically by a more severe pretreatment. However, there are drawbacks: enzymatic degradation would produce GA, which may be toxic to the yeast, and severe pretreatment would drastically lower the yield by causing biomass degradation and release of inhibiting compounds. A fed-batch setup could be a promising alternative to allow high dry-matter concentrations.

\section{Molecular mass distribution of solubilized material}

The molecular mass distribution of the solubilized material was determined after pretreatment (Figure 5A). The 0-0 supernatant did not contain any polymeric material. The absence of water-soluble pectin in SBP may be explained by the prior industrial extraction of saccharose from sugar beet, which involved treatment with calcium hydroxide to precipitate soluble pectins. Three peaks in the small molecular mass region could represent residual saccharose and its hydrolysis products glucose and fructose, or some salts that were solubilized upon hot-water extraction.

The $120^{\circ} \mathrm{C}$ pretreatments both solubilized a polymeric fraction with an average molecular mass of approximately $100 \mathrm{kDa}$, based on the Pullulan molecular mass standards. Samples pretreated with and without sulfuric acid had the same molecular-mass distributions as the samples pretreated with water (data not shown). The average molecular mass of the solubilized material decreased with increasing temperatures from $50 \mathrm{kDa}$ for the $140^{\circ} \mathrm{C}$ pretreatments to oligomeric material of $\leq 1 \mathrm{kDa}$ at $170^{\circ} \mathrm{C}$. Along with the solubilization of carbohydrates, an UV signal at $325 \mathrm{~nm}$ was detected for these populations, indicating the presence of phenols (Figure $5 \mathrm{~B}$ ).

Ferulic acid can be attached to pectic arabinan or galactan $[39,40]$, and could explain the UV signal in the molecular mass range of $\geq 1 \mathrm{kDa}$. The UV signal in the molecular mass range of $\leq 1 \mathrm{kDa}$ could indicate the presence of aromatic degradation products, such as furfural or HMF. The ferulic acid content of the supernatants was determined separately (Table 4 ) and compared with the HPSEC data (Figure 5B). The release of ferulic acid was important for two reasons. Ferulic acid esters present in arabinan and galactan can inhibit the complete degradation of biomass and lower the monosaccharide yields. Secondly, ferulic acid is a high value byproduct in itself, because it is a precursor of aromatic compounds, such as vanillin [41]. With increasing severity of the pretreatment, increasing amounts of ferulic acid were present in the solubilized fractions, which could explain the 

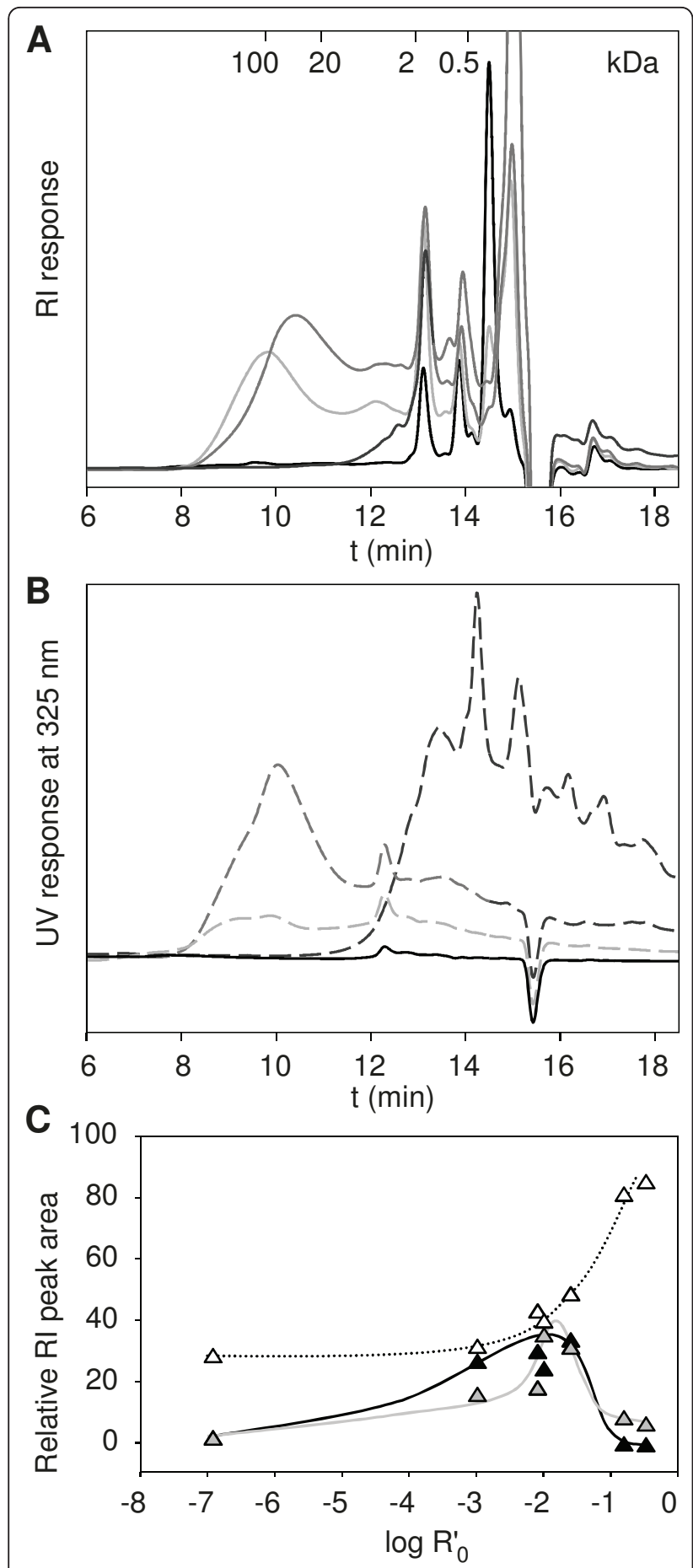

Figure 5 Molecular mass distribution of solubilized material. (A,B) High performance size exclusion chromatography (HPSEC) elution pattern of 0-0 (black), 120-1 (light grey), 140-1 (grey) and 170-1 (dark grey).(A) Solid lines = RI signal, (B) dashed lines = UV signal at $325 \mathrm{~nm}$. (C) Logarithm of the combined severity factor $\left(\log R_{0}^{\prime}\right)$ vs. the relative RI peak area. Black triangles = peak area of the time interval 7.0 to 11.0 minutes, grey triangles $=$ peak area of the time interval 11.0 to 13.0 minutes, white triangles $=$ peak area of the time interval 13.0 to 15.2 minutes. Lines show trends.
Table 4 Ferulic acid (FA) yield in solubilized material after pretreatment

\begin{tabular}{lrr}
\hline Sample & $\mathrm{FA}^{\mathbf{a}} \mathbf{n m o l} / \mathbf{m g}$ & $\mathrm{FA}^{\mathbf{b}}, \%$ \\
\hline $0-0$ & 0 & 0 \\
$120-0$ & 6 & 6 \\
$120-1$ & 9 & 11 \\
$140-0$ & 20 & 31 \\
$140-1$ & 21 & 38 \\
$170-0$ & 36 & 61 \\
$170-1$ & 34 & 59 \\
\hline
\end{tabular}

${ }^{a}$ Absolute amount of solubilized ester-linked FA

${ }^{b}$ Relative amount of solubilized ester-linked FA.

high UV absorption of the $140^{\circ} \mathrm{C}$ fractions at 10 minutes and the UV absorption of the $170^{\circ} \mathrm{C}$ samples in the oligomeric range at 13 to 15 minutes during the reaction.

An optimal pretreatment does not necessarily hydrolyze the insoluble material to monosaccharides and oligosaccharides, which are more prone to chemical destruction than are polymers. The molecular mass distributions of the supernatants indicated that the relative proportions of the high and average molecular mass material increased up to $\log R_{0}^{\prime}=-1.65$ (sample 140-1), whereas they strongly decreased for pretreatments with $\log R_{0}^{\prime} \geq-0.86$ (Figure $5 \mathrm{C})$. In turn, the amount of each compound eluting in the monomeric and oligomeric range increased with increasing severity. These results show, together with the decomposition (Figure 2; Figure 3D) that pretreatments with log $R_{0}^{\prime} \geq-0.86$ favored chemical destruction of sugars and were too severe when aiming for a maximum yield of fermentable sugars.

\section{Enzymatic degradation of pretreated SBP pellets}

For bioethanol production, the aim is to ferment a wide range of monosaccharides. Besides hexose-fermenting yeast strains (mainly glucose and fructose but also galactose), several yeast strains that are able to ferment pentoses have been described in the literature [42]. The efficient enzymatic degradation of sugar-beet arabinan has already been reported [19]. To verify the influence of the pretreatment on enzymatic digestibility of cellulose, we degraded pretreated SBP pellets with an experimental enzyme mixture (C1-G1) that is rich in cellulases and xylanases (Table 2). This mixture has been designed for the degradation of wheat bran, but also has endogalactanase and endoarabinanase activities (Table 2). It is almost devoid of polygalacturonase/pectate lyase activity (Table 2).

Polygalacturonase deficiency could be beneficial for a subsequent yeast fermentation because high concentrations of weak acids have a negative effect on yeast viability, and could therefore lower ethanol production [10]. We were able to recover sugar-beet polygalacturonides after fermentation. These polygalacturonides can be used to produce 
pectin-derived oligosaccharides enzymatically. Pectinderived oligosaccharides have been shown to have a number of beneficial biological effects, including prebiotic activity [43-45]. Alternatively, enzymatically released GA may be fermented to ethanol by bacteria [46].

We analyzed the supernatants of the enzyme digested pellets for their monomer sugar content, and calculated the yield for each individual sugar (Table 5). Glucose was the main monosaccharide released by C1-G1. Besides glucose, up to $4 \%$ of cellobiose was present after 48 hours, indicating insufficient $\beta$-glucosidase activity (data not shown). Glucose yields increased from $17 \%$ for the $0-0$ pellet up to $91 \%$ for the $140-1$ pellet, then dropped to 61 to $65 \%$ for the $170^{\circ} \mathrm{C}$ pellets. This pattern was confirmed by analysis of the sugar composition of the pellets after 48 hours of incubation with the enzyme (data not shown). A comparison of the glucose content of the starting material with that of the supernatants and pellets after enzymatic degradation confirmed that no glucose was destroyed. The reduced glucose release after the $170^{\circ} \mathrm{C}$ pretreatments could indicate enzyme inhibition, or a structural change in the cell-wall complex that made cellulose less accessible.

There was no GA release and only a small release of galactose seen. Some arabinose monosaccharides were released, reaching a yield of up to $29 \%$ based on the arabinose originally present in the pellet fraction. Monomer yields of arabinose and galactose could be further increased by the addition of arabinofuranosidases and galactosidases, respectively, because arabinose oligomers up to DP 6 and galactobiose were detected by HPAEC. Taken together, our data indicate that C1-G1 was able degrade up to $62 \%$ of all neutral sugars present in the pretreated pellets to monomers, including $91 \%$ of glucose originating from cellulose. The time-dependent enzymatic hydrolysis of cellulose from pretreated pellets was also studied (Figure 6). In general, cellulose hydrolysis was faster for the samples that were pretreated with sulfuric acid. For samples 120-1 and 140-1, the endpoint

Table 5 Yields of individual monosaccharides ending up in the supernatant after enzyme incubation for $\mathbf{4 8}$ hours of pretreated pellets with C1-G1 enzyme mixture $(1 \% \mathrm{w} / \mathrm{w})^{\mathrm{a}}$

\begin{tabular}{lrrrrrr}
\hline Sample & Ara & Gal & Glc & Xyl & Man & Total $^{\mathbf{b}}$ \\
\hline $0-0$ & 6 & 3 & 17 & 6 & 3 & 9 \\
$120-0$ & 12 & 4 & 75 & 14 & 20 & 36 \\
$120-1$ & 29 & 4 & 83 & 21 & 23 & 52 \\
$140-0$ & 24 & 3 & 86 & 21 & 23 & 58 \\
$140-1$ & 24 & 2 & 91 & 18 & 26 & 62 \\
$170-0$ & 0 & 0 & 61 & 19 & 9 & 54 \\
$170-1$ & 0 & 0 & 65 & 22 & 9 & 59
\end{tabular}

${ }^{a}$ Values in \% of total amount of individual sugar present in the pellets.

${ }^{b}$ Yield of all monosaccharides (\% of total neutral sugar present in the pellets.

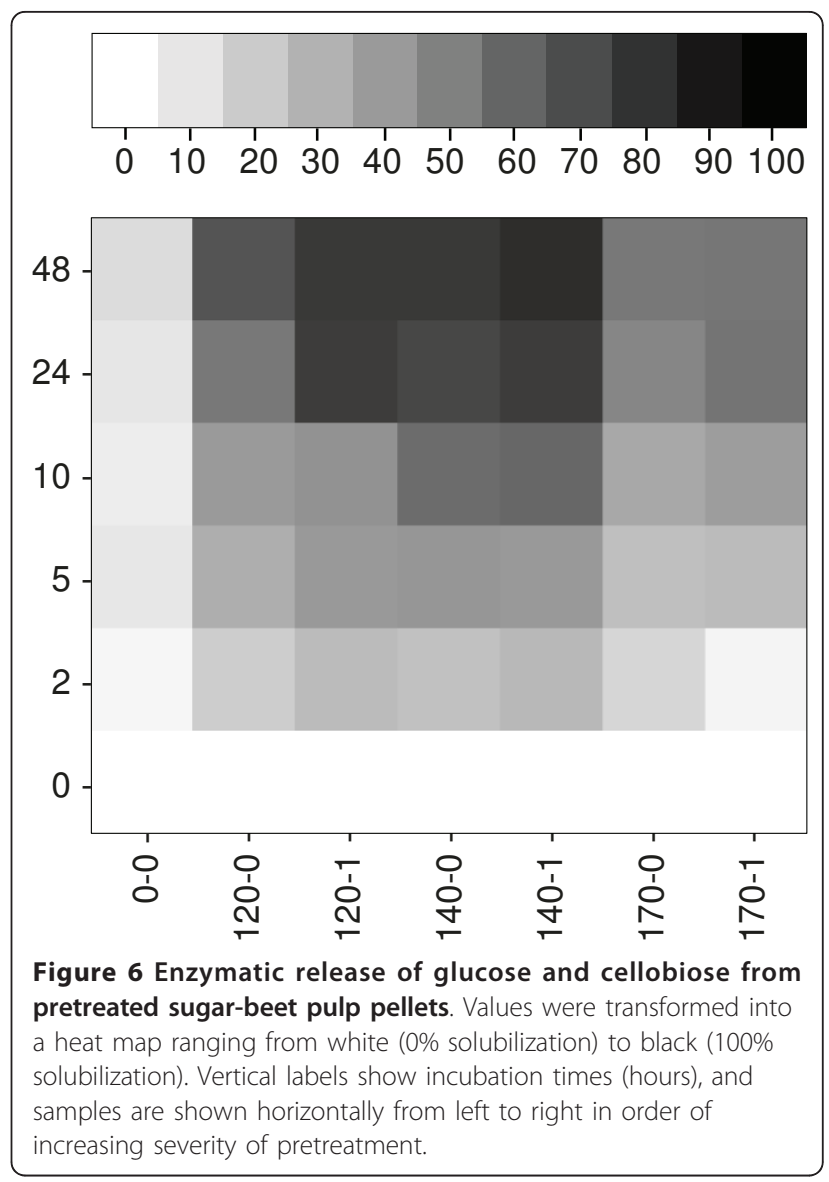

was almost reached after 24 hours, whereas $120-0$ and 140-0 required 48 hours for maximal hydrolysis (Figure 6). Ultimately, more sugars were hydrolyzed with pretreatment 140-0 and subsequent enzyme digestion, because slightly higher losses occur at 140-1 (Figure $3 \mathrm{D})$. Therefore, pretreatment 140-0 was the optimal pretreatment for the release of glucose.

\section{Total solubilization after pretreatment and enzymatic digestion}

Upon enzyme digestion of the pretreated pellets, the pellets of all pretreated samples were enriched in protein content from $8 \% \mathrm{w} / \mathrm{w}$ up to $25 \% \mathrm{w} / \mathrm{w}$, indicating that the main part of the proteins remained insoluble during pretreatment, whereas their sugar content strongly decreased (data not shown). The influence of the pretreatment and enzymatic digestion on the solubility of each individual sugar was analyzed for all samples (Figure 7; see Additional file 3, Table s2). After pretreatment, the minor constituents rhamnose and galactose were effectively solubilized at $170^{\circ} \mathrm{C}$, whereas the major constituents arabinose and UA (mainly GA) gave only a $60 \%$ yield at $140^{\circ} \mathrm{C}$ and were destroyed at higher temperatures (Figure 7A). 


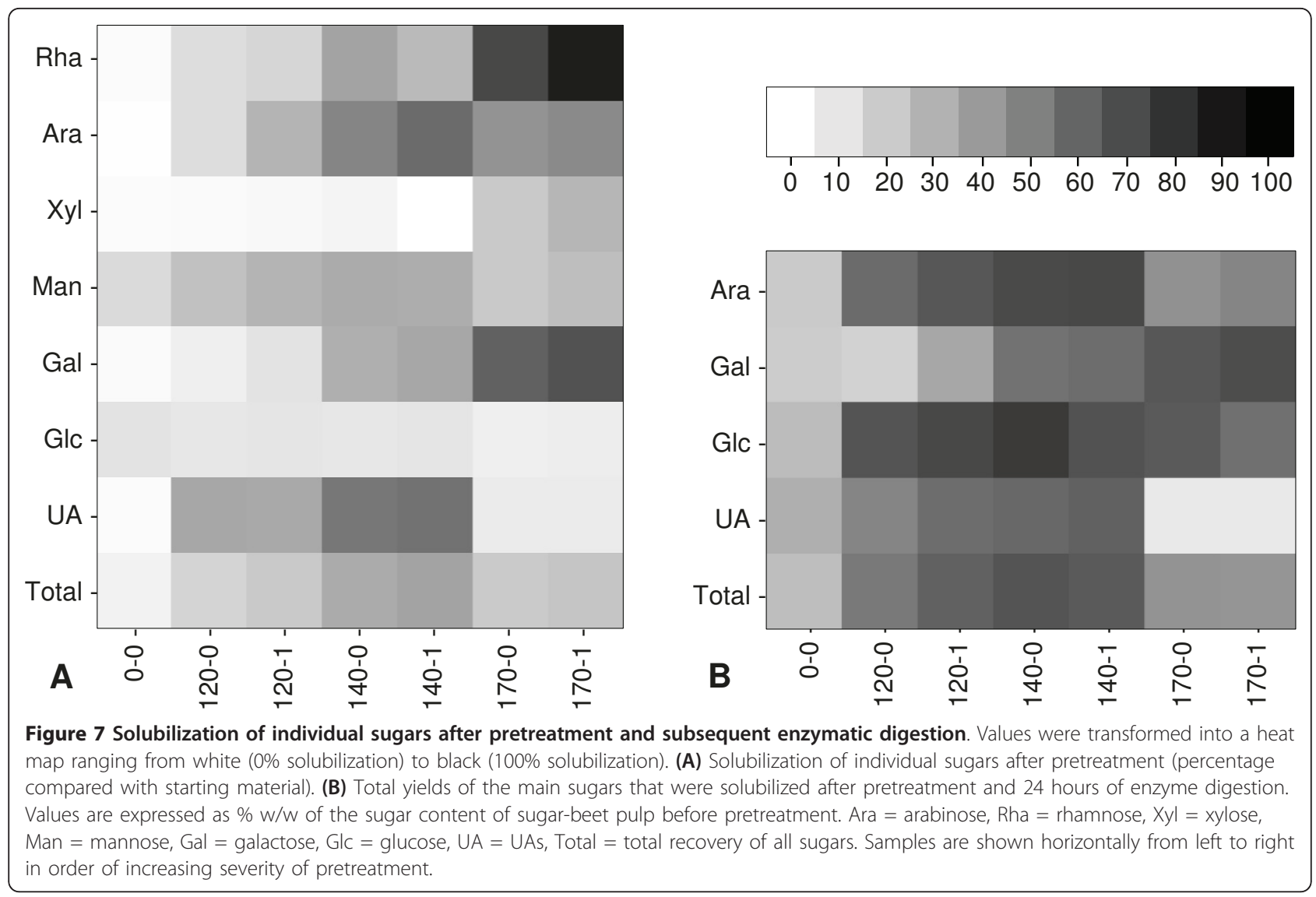

The total carbohydrate solubilization was calculated as the sum of all material solubilized after pretreatment plus the material solubilized from the pellet after enzymatic digestion (Figure 7B). The solubilization was inversely correlated with increasing severity, and ranged from $34 \%$ for the $0-0$ digest to $80 \%$ for the $140-0$ digest. The pectin-associated sugars arabinose, galactose and GA had 85,70 and $75 \%$ solubilization, respectively, in sample 140-1. Mild pretreatments up to $\log R_{0}^{\prime}=-1.65$ generally enhanced the enzymatic degradation of cellulose from SBP (Figure 7B). C1-G1 also released material of high molecular mass from the pellets of samples 0-0, 120-0 and 120-1 (determined by HPSEC analysis, data not shown). It is likely that the solubilized material was pectin, because after enzyme incubation for 48 hours, the pellets had a strong reduction in their UA content (data not shown). Pectin can be solubilized either by the degradation of the cellulose network or by the removal of arabinan sidechains linking it to cellulose [28].

\section{Conclusions}

We found that the combination of a mild hydrothermal pretreatment at $140^{\circ} \mathrm{C}$ with subsequent enzymatic digestion allowed fast and efficient hydrolysis of SBP. More than $90 \%$ of all cellulose could be hydrolyzed within 24 hours, using lower enzyme levels than those reported in earlier studies. In contrast to the chemical pretreatments described in literature, mild pretreatments up to $140^{\circ} \mathrm{C}$ did not result in a loss of biomass or in production of any compounds toxic to yeast. If the pretreatment process were to be implemented in a sugar factory, the pulp would already be at $70^{\circ} \mathrm{C}$ after saccharose extraction, and the exhaust heat of the lime ovens could be used in part for the heating of the SBP up to $140^{\circ} \mathrm{C}$. The mild pretreatments did not lower the WBC of beet pulp and are therefore unsuitable for increasing the drymatter content of SBP. Hence, to reduce transportation, pretreated SBP should be fermented in the vicinity of the sugar factory. A fed-batch reactor setting could allow a reasonably high ethanol concentration.

\section{Additional material}

Additional file 1: Figure S1. Pressure and temperature curves of a $170^{\circ} \mathrm{C}$ pretreatment. Black line $=$ temperature $\left({ }^{\circ} \mathrm{C}\right)$, grey line $=$ pressure (bar).

Additional file 2: Table S1: Molar sugar composition of pretreated samples.

Additional file 3: Table S2: Volumetric concentrations of the main sugars present in sugar-beet pulp obtained after pretreatment and 24 hours of enzyme digestion. ${ }^{2}$ Volumetric concentrations of arabinose 
(Ara), galactose (Gal), glucose (Glc) and uronic acid s (UA) present in the supernatant fraction $(\mathrm{g} / \mathrm{l}){ }^{\mathrm{b}}$ Amount of sugars $(\mathrm{g})$ present in $50 \mathrm{~g}$ starting material.

\section{Acknowledgements}

We thank David van Eylen (Royal Nedalco/C5 Yeast Company, Bergen op Zoom, The Netherlands) for help with the pretreatments. Mirjam Kabel (Royal Nedalco/C5 Yeast Company) is gratefully acknowledged for valuable discussion, and Sandra Hinz (Dyadic Netherlands) for the provision and characterization of the C1-G1 enzyme mixture. The work was financed in part by the Dutch Ministry of Economic Affairs via an EOS-LT grant (http://www.agentschapnl.nl).

\section{Authors' contributions}

SK and HAS designed and coordinated the study. SK carried out the experiments and prepared the manuscript. HAS and HG contributed to the manuscript preparation. All authors read and approved the final version of the manuscript.

\section{Competing interests}

The author declares that they have no competing interests.

Received: 11 February 2011 Accepted: 31 May 2011

Published: 31 May 2011

\section{References}

1. McCready RM: Polysaccharides of sugar beet pulp. A review of their chemistry. J Am Soc Sugar Beet 1966, 14:260-270.

2. Kelly P: Sugar beet pulp - A review. Anim Feed Sci Tech 1983, 8:1-18.

3. Voragen AGJ, Oosterveld A, Schols HA, Beldman G: Pectic substances from sugar beet pulp: extraction and fractionation, structural features, functional properties and enzymic modification. Transformation and modification of carbohydrates, Proc of 4th Int Workshop on carbohydrates as organic raw materials Vienna, Austria; 1997, 29.

4. Bettiga M, Bengtsson O, Hahn-Hägerdal B, Gorwa-Grauslund MF: Arabinose and xylose fermentation by recombinant Saccharomyces cerevisiae expressing a fungal pentose utilization pathway. Microb Cell Fact 2009, 8.

5. Alvira P, Tomás-Pejó E, Ballesteros M, Negro MJ: Pretreatment technologies for an efficient bioethanol production process based on enzymatic hydrolysis: a review. Bioresource Technol 2010, 101:4851-4861.

6. Abatzoglou N, Chornet E, Belkacemi K, Overend RP: Phenomenological kinetics of complex systems: the development of a generalized severity parameter and its application to lignocellulosics fractionation. Chem Eng Sci 1992, 47:1109-1122.

7. Chum HL, Johnson DK, Black SK, Overend RP: Pretreatment-catalyst effects and the combined severity parameter. Appl Biochem Biotech 1990, 24-25:1-14.

8. Palmqvist $E$, Hahn-Hägerdal B: Fermentation of lignocellulosic hydrolysates. II: Inhibitors and mechanisms of inhibition. Bioresource Technol 2000, 74:25-33.

9. Banerjee N, Bhatnagar R, Viswanathan L: Inhibition of glycolysis by furfural in Saccharomyces cerevisiae. Eur J Appl Microbiol 1981, 11:226-228.

10. Rottenberg H, Fleischer S, Packer $L$ : The measurement of membrane potential and [Delta]pH in cells, organelles, and vesicles. Method Enzymol 1979, 55:547-569.

11. Palmqvist $E$, Hahn-Hägerdal B: Fermentation of lignocellulosic hydrolysates. I: Inhibition and detoxification. Bioresource Technol 2000, 74:17-24.

12. Beldman G, Rombouts FM, Voragen AGJ, Pilnik W: Application of cellulase and pectinase from fungal origin for the liquefaction and saccharification of biomass. Enzyme Microb Technol 1984, 6:503-507.

13. Chamy R, lllanes A, Aroca G, Nunez L: Acid hydrolysis of sugar beet pulp as pretreatment for fermentation. Bioresource Technol 1994, 50:149-152.

14. Martínez M, Gullón B, Schols HA, Alonso JL, Parajó JC: Assessment of the production of oligomeric compounds from sugar beet pulp. Ind Eng Chem Res 2009, 48:4681-4687.

15. Micard V, Renard CMGC, Thibault JF: Influence of pretreatments on enzymic degradation of a cellulose-rich residue from sugar-beet pulp. Lebensm Wiss Technol 1997, 30:284-291.
16. Kabel MA, Bos G, Zeevalking J, Voragen AGJ, Schols HA: Effect of pretreatment severity on xylan solubility and enzymatic breakdown of the remaining cellulose from wheat straw. Bioresource Technol 2007, 98:2034-2042.

17. Walseth CS: Occurrence of cellulase in enzyme preparation from microorganism. TAPPI 1952, 35:228-233.

18. Lever M: A new reaction for colorimetric determination of carbohydrates. Anal Biochem 1972, 47:273-279.

19. Kühnel S, Hinz SWA, Pouvreau L, Visser J, Schols HA, Gruppen H: Chrysosporium lucknowense arabinohydrolases efficiently degrade sugar beet arabinan. Bioresource Technol 2010, 101:8300-8307.

20. Hinz SWA, Pouvreau L, Joosten R, Bartels J, Jonathan MC, Wery J, Schols HA Hemicellulase production in Chrysosporium lucknowense C1. J Cereal Sci 2009, 50:318-323.

21. Englyst HN, Cummings JH: Simplified method for the measurement of total non-starch polysaccharides by gas-liquid chromatography of constituent sugars as alditol acetates. Analyst 1984, 109:937-942.

22. Ahmed ERA, Labavitch JM: A simplified method for accurate determination of cell wall uronide content. J Food Biochem 1978, 1:361-365.

23. Appeldoorn MM, Kabel MA, van Eylen D, Gruppen H, Schols HA: Characterisation of oligomeric structures from corn fiber resistant towards pretreatment and simultaneous saccharification and fermentation. J Agric Food Chem 2010, 58:11294-11301.

24. Conrad CM: Decarboxylation studies on pectins and calcium pectates. J Am Chem Soc 1931, 53:1999-2003.

25. Dunlop AP: Furfural formation and behavior. Ind Eng Chem 1948, 40:204-209.

26. Zweifel G, Deuel H: Über die Decarboxylierung von Galakturonsäure mit Schwermetallionen. Helv Chim Acta 1956, 39:662-667.

27. Martínez M, Gullón B, Yáñez R, Alonso JL, Parajó JC: Kinetic assessment on the autohydrolysis of pectin-rich by-products. Chem Eng J 2010, 162:480-486.

28. Zykwinska AW, Ralet MC, Garnier CD, Thibault JF: Evidence for in vitro binding of pectin side chains to cellulose. Plant Physiol 2005, 139:397-407.

29. Shallenberger RS, Mattick LR: Relative stability of glucose and fructose at different acid pH. Food Chem 1983, 12:159-165.

30. van Dam HE, Kieboom APG, van Bekkum H: The conversion of fructose and glucose in acidic media: formation of hydroxymethylfurfural. Starch 1986, 38:95-101.

31. Ulbricht RJ, Northup SJ, Thomas JA: A review of 5-hydroxymethylfurfural (HMF) in parenteral solutions. Fund Appl Toxicol 1984, 4:843-853.

32. Voragen AGJ, Schols HA, Pilnik W: Non-enzymatic browning of oligogalacturonides in apple juice models. Z Lebensm Unters Forsch A 1988, 187:315-320.

33. Einhorn-Stoll $U$, Kunzek $H$ : The influence of the storage conditions heat and humidity on conformation, state transitions and degradation behaviour of dried pectins. Food Hydrocolloid 2009, 23:856-866.

34. Hurd CD, Isenhour LL: Pentose reactions. I. Furfural formation. J Am Chem Soc 1932, 54:317-330

35. Reiter WD: Biosynthesis and properties of the plant cell wall. Curr Opin Plant Biol 2002, 5:536-542.

36. Bertin C, Rouau X, Thibault JF: Structure and properties of sugar beet fibres. J Sci Food Agric 1988, 44:15-29.

37. Rouau X, Bertin C, Thibault JF: Characterization and enzymic degradation of sugar beet fibres. Food Hydrocolloid 1987, 1:439-443.

38. Gruppen H, Kormelink FJM, Voragen AGJ: Enzymic degradation of waterunextractable cell wall material and arabinoxylans from wheat flour. $J$ Cer Sci 1993, 18:129-143.

39. Guillon F, Thibault JF, Rombouts FM, Voragen AGJ, Pilnik W: Enzymic hydrolysis of the "hairy" fragments of sugar-beet pectins. Carbohyd Res 1989, 190:97-108.

40. Rombouts FM, Thibault JF: Enzymic and chemical degradation and the fine structure of pectins from sugar-beet pulp. Carbohyd Res 1986, 154:189-203

41. Mathew S, Abraham TE: Ferulic acid: An antioxidant found naturally in plant cell walls and feruloyl esterases involved in its release and their applications. Crit Rev Biotechnol 2004, 24:59-83.

42. van Maris AJA: Efficient ethanol production from arabinose. Ind Bioproc 2007, 29:9-10. 
43. Gullón P, Gullón B, Moure A, Alonso JL, Domínguez H, Parajó JC: Manufacture of Prebiotics from Biomass Sources. Prebiotics and Probiotics Science and Technology 2009, 535-589.

44. Hotchkiss AT, Olano-Martin E, Grace WE, Gibson GR, Rastall RA: Pectic oligosaccharides as prebiotics.Edited by: Eggleston G, Côté GL. American Chemical Society, Washington, D. C., USA; 2003:849:54-62.

45. Mandalari G, Nueno Palop C, Tuohy K, Gibson GR, Bennett RN, Waldron KW, Bisignano G, Narbad A, Faulds CB: In vitro evaluation of the prebiotic activity of a pectic oligosaccharide-rich extract enzymatically derived from bergamot peel. Appl Microbiol Biot 2007, 73:1173-1179.

46. Doran JB, Cripe J, Sutton M, Foster B: Fermentations of pectin-rich biomass with recombinant bacteria to produce fuel ethanol. Appl Biochem Biotechn 2000, 84-86:141-152.

47. Overend RP, Chornet E, Gascoigne JA: Fractionation of lignocellulosics by steam-aqueous pretreatments [and discussion]. Phil Trans R Soc Lond A 1987, 321:523-536

doi:10.1186/1754-6834-4-14

Cite this article as: Kühnel et al: Aiming for the complete utilization of sugar-beet pulp: Examination of the effects of mild acid and hydrothermal pretreatment followed by enzymatic digestion.

Biotechnology for Biofuels 2011 4:14.

\section{Submit your next manuscript to BioMed Central} and take full advantage of:

- Convenient online submission

- Thorough peer review

- No space constraints or color figure charges

- Immediate publication on acceptance

- Inclusion in PubMed, CAS, Scopus and Google Scholar

- Research which is freely available for redistribution

Submit your manuscript at www.biomedcentral.com/submit 